\title{
Exercise Capacity of Patients with Type 2 Diabetes: A Mini Review of Clinical Findings
}

\author{
Hisayo Yokoyama ${ }^{1 *}$ and Masanori Emoto ${ }^{2}$ \\ ${ }^{1}$ Research Center for Urban Health and Sports, Osaka City University, 3-3-138, Sugimoto, Sumiyoshi-ku, Osaka, Japan \\ ${ }^{2}$ Metabolism, Endocrinology and Molecular Medicine, Osaka City University Graduate School of Medicine, Osaka, Japan
}

\begin{abstract}
Received: July 07, 2015; Accepted: August 15, 2015; Published: September 11, 2015
*Corresponding author: Hisayo Yokoyama, Research Center for Urban Health and Sports, Osaka City University,3-3-138,Sugimoto, Sumiyoshi-ku, Osaka, 558-8585, Japan, Tel: +81-6-6605-2947; Fax: +81-6-6605-2947; E-mail: yokoyama@sports.osaka-cu.ac.jp
\end{abstract}

\begin{abstract}
In this mini review, we aimed to clarify the contributors to impaired cardiorespiratory fitness in patients with type 2 diabetes and to discuss how to improve cardiorespiratory fitness in this population. Patients with type 2 diabetes have low cardiorespiratory fitness even if the duration of their disease has been short and they have had no apparent diabetic complications or evident cardiovascular disease Impaired cardiorespiratory fitness results in a sedentary lifestyle and an increased risk of cardiovascular and all-cause mortality. Conversely, physical inactivity exacerbates low cardiorespiratory fitness in this population. The pathognomonic factors in patients, such as impaired endothelial dysfunction and vascular conductance or mitochondrial dysfunction, contribute to reduced oxygen utilization in working muscles, which causes abnormal oxygen uptake kinetics during exercise. Clinically negligible diabetic cardiomyopathy, including left ventricular diastolic dysfunction and cardiac autonomic neuropathy, may also slow oxygen uptake kinetics and affect the cardiorespiratory fitness of a patient with diabetes. Although habitual exercise itself is the most efficient way to improve cardiorespiratory fitness, studies have suggested that patients with diabetes could benefit from thiazolidinedione to augment cardiorespiratory fitness.
\end{abstract}

Keywords: Cardiorespiratory Fitness; Type 2 Diabetes; Oxygen Uptake Kinetics; Sedentary Lifestyle; Thiazolidinedione

\section{Introduction}

Clinical investigations have revealed that patients with type 2 diabetes exhibit poor cardiorespiratory fitness compared with their age-matched, non-diabetic counterparts [1,2]. It is well known that poor cardiorespiratory fitness causes an increased risk of cardiovascular and all-cause mortality in this population $[3,4]$. Therefore, it is important to understand the causal factors associated with impaired cardiorespiratory fitness for appropriate management of the disease. In the present mini review, we appraise previous reports and aim to clarify the contributors to impaired cardiorespiratory fitness in patients with type 2 diabetes. We also discuss methods of improving cardiorespiratory fitness in this population.

\section{A Small Number of Patients with Type 2 Diabetes Regularly Exercise}

Exercise is an established therapeutic strategy for type 2 diabetes. Regular exercise results in good glycemic control, weight loss, and an improvement in insulin sensitivity. In a joint position statement from the American Diabetes Association and the American College of Sports Medicine in 2010, exercise was recommended for most people with type 2 diabetes. Patients are particularly encouraged to engage in aerobic exercise at least three times per week. This entails a minimum period of $150 \mathrm{~min}$ a week combined with resistance training at least twice a week [5].

However, patients with diabetes are often sedentary. According to a survey of the US population including 23,283 people in 2003 , compared with $58 \%$ of adults without diabetes, only $39 \%$ of adults with diabetes were physically active, i.e., engaged in more than 30 min of moderate or vigorous physical activity at least three times a week [6]. A UK study also revealed that two-thirds of patients with diabetes had not participated in any physical activity in the two weeks before the study [7]. Among these "inactive" patients, younger ( $<60$ years) patients stated that a lack of spare time or local facilities were the main barriers to exercise. In addition, they were less likely to exercise if they were tired.

\section{Low Cardiorespiratory Fitness in the Type 2 Diabetic Population}

It is well known that patients with type 2 diabetes have low cardiorespiratory fitness, as evaluated by peak oxygen consumption (peak $\mathrm{VO}_{2}$ ) or peak metabolic equivalents (METs) during a maximum graded exercise test [1,2]. Even if these patients have had a short duration of disease and no obvious diabetic complications or cardiovascular diseases, peak $\mathrm{VO}_{2}$ of the patients was reduced by $12 \%-15 \%$ compared with the reference level of age-matched healthy subjects [8]. The reduced cardiorespiratory fitness in this population will certainly cause fatigability or pain during exercise and further augment their sedentary lifestyle. Such physical inactivity conversely exacerbates low cardiorespiratory fitness in a vicious cycle. However, it seems that there are other contributors because the cardiorespiratory fitness of patients with diabetes was reported to be approximately $10 \%$ lower than that of their non-diabetic 
counterparts, who had a similar level of physical activity [1]. In the following section, we focus on possible contributors to impaired cardiorespiratory fitness in patients with type 2 diabetes, other than physical inactivity.

\section{Why are patients with type 2 diabetes intolerant of exercise?}

\section{Impaired Oxygen Uptake Kinetics}

Besides physical inactivity, various pathognomonic factors of type 2 diabetes seem to contribute to low cardiorespiratory fitness, although the exact pathological mechanisms have not been fully resolved. One candidate is abnormal oxygen uptake kinetics, i.e., there is a slower rate of change in $\mathrm{VO}_{2}$ in response to the onset of constant-load exercise, which seems to affect cardiorespiratory fitness in patients with type 2 diabetes. This kinetics generally reflects the ability to transport oxygen to the working muscles and to utilize delivered oxygen for oxidative energy metabolism. Slow oxygen uptake kinetics has also been reported to negatively correlate with peak $\mathrm{VO}_{2}$ [9]. Many previous studies have demonstrated that oxygen uptake kinetics in steadystate exercise were reduced by an average of $35 \%$ in those who had diabetes, especially younger ( $<60$ years) female patients [8]. The impaired transport of oxygen to working muscles is considered to be mainly due to the insufficiency of muscular hyperemia during exercise because of impaired endothelial function and vascular conductance, traits that are commonly seen in patients with diabetes [10]. Furthermore, mitochondrial depletion and dysfunction in skeletal muscle cells may also reduce local oxygen utilization [8]. These factors are possibly related to the impaired oxidative metabolism in skeletal muscles and result in slow oxygen uptake kinetics during exercise in this population.

\section{Subclinical Left Ventricular (LV) Dysfunction}

Subclinical LV dysfunction may be another contributor to slow oxygen uptake kinetics and impaired cardiorespiratory fitness. Diabetic cardiomyopathy is defined as the presence of idiopathic myocardial involvement directly due to diabetes, and its presence is independent of the co-existence of ischemic, hypertensive, or valvular heart disease [11]. In most cases, LV diastolic dysfunction is the earliest manifestation of diabetic cardiomyopathy. As reported in a study with healthy subjects [12], previous reports have demonstrated that LV diastolic dysfunction is defined as reduced early diastolic velocity evaluated using Doppler echocardiography and is associated with decreased peak $\mathrm{VO}_{2}$ in type 2 diabetes without apparent coronary artery disease $[13,14]$. A reduction in LV end-diastolic volume restrains cardiac output during exercise, according to the Frank-Starling law. In addition, LV diastolic dysfunction increases pulmonary artery wedge pressure followed by relative pulmonary congestion, limiting peak $\mathrm{VO}_{2}$ in the graded exercise test [13]. It has also been suggested that cardiac autonomic neuropathy, considered as one of the pathophysiologic mechanisms for diabetic cardiomyopathy, represented by poor heart rate variability or impaired heart rate recovery (HRR) from maximum heart rate in a graded exercise test may have contributed to the impaired cardiorespiratory fitness in patients with type 2 diabetes $[14,15]$. We examined the association of HRR with peak METs in a maximum graded exercise test in 74 diabetic patients with poorly-controlled glycemic profiles (37 males, $62.2 \pm 9.2$ (SD) years; duration of diabetes, $8.9 \pm 7.9$ years; HbA1c, $9.3 \pm 1.9 \%$ ), who were admitted to PL hospital, Tondabayashi City, Osaka (unpublished data). As shown in Figure 1, HRR showed a statistically significant positive correlation with peak METs. Furthermore, in multiple regression analysis, HRR was found to be a strong determinant for exercise capacity and was independent of age and gender (Table 1). Our findings are consistent with those of aforementioned reports $[14,15]$. However, a more recent study by Gürdal et al. reported that peak $\mathrm{VO}_{2}$ in the maximum graded exercise test showed no statistically significant difference irrespective of the presence or absence of $\mathrm{LV}$ diastolic dysfunction in patients with type 2 diabetes and who had no apparent coronary artery disease [16]. Sacreet et al. also reported that a 6-month exercise intervention successfully increased peak $\mathrm{VO}_{2}$ by $11 \%$ in patients with type 2 diabetes. However, this was not accompanied by significant improvement in the HRR or LV diastolic function [17]. The influence of LV diastolic dysfunction on cardiorespiratory fitness possibly varies depending on the extent of pre-existing subclinical myocardial damage. This is because cardiac rehabilitation for patients suffering from more severe myocardial functional abnormalities or heart failure with reduced LV ejection fraction culminates in an improvement in both $\mathrm{LV}$ function and peak $\mathrm{VO}_{2}[18]$. However, the mechanism of this relationship remains to be clarified.

\section{Insulin Resistance and Poor Glycemic Control}

Several reports have shown that insulin resistance, evaluated by an euglycemic-hyperinsulinemic clamp or a homeostasis model assessment was negatively correlated with cardiorespiratory fitness in this population $[19,20]$. Insulin resistance not only plays an important role in hyperglycemia in patients with type 2 diabetes but is also associated with all of the aforementioned pathological conditions such as myocardial

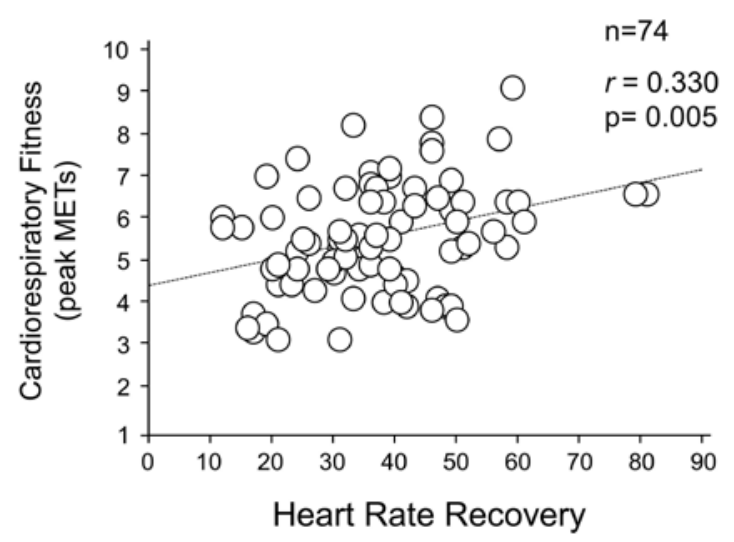

Figure 1: Correlation between heart rate recovery and cardiorespiratory fitness (peak METs) in the maximum graded exercise test. Heart rate recovery was significantly positively correlated with peak METs ( $\mathrm{r}$ $=0.330, p=0.005$ ).

Abbreviation: METs, metabolic equivalents 
Table 1: Multiple regression analysis for cardiorespiratory fitness.

\begin{tabular}{|l|c|c|}
\hline & $\boldsymbol{B}$ & $\boldsymbol{p}$ \\
\hline Age & -0.272 & 0.018 \\
\hline $\begin{array}{l}\text { Gender (male=1, } \\
\text { female=0) }\end{array}$ & 0.265 & 0.015 \\
\hline Heart Rate Recovery & 0.232 & 0.042 \\
\hline $\begin{array}{l}\mathrm{R}^{2}=0.504, p<0.001 \\
\mathrm{~B}_{\text {are standard coefficient of each independent variable. }}\end{array}$ \\
$\mathrm{R}^{2}$ is the coefficient of determination.
\end{tabular}

systolic/diastolic dysfunction [21], endothelial dysfunction [22], and mitochondrial disability in skeletal muscle [23]. Recently, impaired cardiac insulin signaling, as well as cardiac mitochondrial depletion and dysfunction, has attracted much attention for playing an important role in the pathogenesis of diabetic cardiomyopathy [24]. However, it has not been fully elucidated whether insulin resistance in skeletal and cardiac muscles is synchronously acquired. Therefore, the presence of insulin resistance may result in reduced peak $\mathrm{VO}_{2}$ in the maximum graded exercise test. In contrast, a few reports have suggested an association between insufficient glycemic control and impaired cardiorespiratory fitness [14] and restored cardiorespiratory fitness after the improvement of hyperglycemia by insulin therapy [25]. Meanwhile, other investigators failed to detect any significant correlation between the two. Although the reason for these inconsistent observations is not clear, atrophy in the proximal muscles or the tendency towards dehydration is shared by patients with type 2 diabetes who have poor control over the disease, and this potentially contributes to the reduced cardiorespiratory fitness.

\section{Poor prognosis in type 2 diabetes with reduced cardiorespiratory fitness}

It is known that a sedentary lifestyle and impaired cardiorespiratory fitness are strong predictors of cardiovascular and all-cause mortality in a healthy population [26]; unsurprisingly, the same is true for patients with type 2 diabetes. This is because cardiorespiratory fitness reflects other factors (including metabolic profile, cardiovascular function, or other complicated medical conditions), which have already been established as predictors of survival in these patients. From the results of several prospective cohort studies, 1-peak MET increments in the maximal exercise test reduced the adjusted risk of all-cause mortality by $14 \%-25 \%$ in this population $[3,4]$. It has also been reported that high cardiorespiratory fitness equally ameliorated cardiovascular disease-related mortality in patients with type 2 diabetes who were overweight or obese, compared with that observed in their normal-weight counterparts [27]. Therefore, improving cardiorespiratory fitness takes priority over all clinical treatment plans in the management of type 2 diabetes.

\section{How to improve cardiorespiratory fitness in patients with type 2 diabetes?}

Engaging in regular exercise or physical activity is the best way to augment cardiorespiratory fitness. To date, many investigators have shown that cardiorespiratory fitness is principally improved by aerobic exercise in patients with type 2 diabetes [28]. A meta-analysis of nine RCTs concluded that aerobic training of 3.4 sessions/week, $49 \mathrm{~min} / \mathrm{session}$ for 20 weeks on average with an intensity of $50 \%-75 \%$ of peak $\mathrm{VO}_{2}$ resulted in an $11.8 \%$ increase in peak $\mathrm{VO}_{2}$. Furthermore, higher exercise intensities could provide a greater improvement [29]. Nevertheless, as previously mentioned, only a small number of patients practice habitual exercise, and most of them lead a sedentary lifestyle. The investigation into the ProActive cohort over 12 months revealed that male gender and higher cardiorespiratory fitness levels independently predicted significant increase in energy expenditure through physical activity [30]. In other words, patients with poor cardiorespiratory fitness find it extremely hard to continue exercising.

Commitment to exercise by the medical staff, adequate space and equipment, or interesting and stimulating regional programs or interventions, are necessary elements for improving adherence to exercise in these unfit and unmotivated patients. More patients with type 2 diabetes will possibly undertake workouts if counseled to do so. However, counseling by the medical staff unfortunately does not seem to be sufficient. According to the National Health Interview Survey in 2010, 56\% of adults with diabetes in the US had been recommended to commence or continue to perform exercise or physical activity by their physicians or other health professionals in the previous year [31]. Although these patients were more likely to have been advised exercise than those patients who had other chronic diseases, such as hypertension, cardiovascular disease, and cancer, patients were still less likely to attempt exercise.

Regarding the potential pharmacological approaches used to augment cardiorespiratory fitness, thiazolidinedione (TZD), an insulin-sensitizing agent, is known to improve peak $\mathrm{VO}_{2}$ in the graded exercise test in patients with type 2 diabetes $[32,33]$. No human study regarding the effect of pioglitazone on cardiorespiratory fitness is available, although a few reports have shown that pioglitazone improved exercise capacity in diabetic mice [34]. It has been reported that 4-8 $\mathrm{mg} /$ day of rosiglitazone administration increased peak $\mathrm{VO}_{2}$ by $4 \%-7 \%$ in patients with type 2 diabetes [32,33], although a few studies with a relatively short-term intervention period (4-12 weeks) concluded that there was no effect of rosiglitazone therapy on cardiorespiratory fitness [35,36]. Some mechanisms for TZDs and how these improve cardiorespiratory fitness have been suggested. First, TZDs stimulate the differentiation of adipocytes as an agonist for the nuclear hormone receptor proxisome proliferator-activated receptor $\gamma$ (PPAR $\gamma$ ) and for the storage of fatty acids mainly in the subcutaneous adipose tissue [37]. These changes in the adipocyte consequently decrease the level of intramyocellular/ intrahepatic triglycerides and cellular lipotoxicity, which results in improvements in mitochondrial and endothelial functions. In addition, the differentiation of adipocytes increases the secretion of adiponectin, an adipocytokine known to ameliorate insulin resistance. Adiponectin is also known to induce the production 
of nitric oxide from arterial endothelial cells [38]. Kadoglou et al. has demonstrated that the rosiglitazone-induced increase in cardiorespiratory fitness was closely associated with an increase in plasma adiponectin in patients with type 2 diabetes [39]. Furthermore, TZDs as well as exercise are known to activate the skeletal muscle AMP-activated protein kinase (AMPK), which most likely results in an increase in cardiorespiratory fitness through enhanced fat oxidation and mitochondrial biogenesis in the skeletal muscle. However, it should be acknowledged that the administration of TZDs is only an option. Exercise or increased physical activity is a low-cost, simple intervention for the management of diabetes and should be given first priority in the strategy for improving cardiorespiratory fitness. Furthermore, the therapeutic approach using TZDs should be considered on a case-by-case based on the balance of the aforementioned advantages and potential side effects (including increasing serum LDL-cholesterol level and bone fracture) of the drugs as well as the existing patient co-morbidities.

It has been shown that metformin, another activator of AMPK, reduced peak $\mathrm{VO}_{2}$, peak heart rate, and exercise duration in the graded exercise test with healthy, normal-weight subjects [40]. administration of metformin did not affect cardiorespiratory fitness [41]. The inconsistency of TZD and metformin effects on cardiorespiratory fitness may be attributed to the difference in the mechanism of how these two insulin sensitizers activate AMPK. These two drugs share the process of indirect AMPK activation by inhibiting complex 1 of the mitochondrial respiratory chain and by consequently increasing AMP synthesis; specifically, TZDs activate AMPK through the effects of PPAR $\gamma$ to stimulate adiponectin secretion. It remains to be clarified whether the unfavorable effect of metformin on exercise capacity is the same for patients with diabetes or obesity, although a non-randomized, observational study concluded that metformin users with type 2 diabetes had no disadvantage in improving their cardiorespiratory fitness by aerobic or resistance training [42].

\section{Summary}

It is now evident that cardiorespiratory fitness is impaired in patients with type 2 diabetes because of other pathognomonic factors in addition to their sedentary lifestyle. Consequently, impaired cardiorespiratory fitness is associated with poor prognosis in this population. To stimulate more patients in performing regular exercise, patients and medical staff ought to find opportunities to discuss the positive aspects of exercise. In addition, optimal exercise programs should be delivered according to the individual physical strength, medical concerns, and convenience. Patients can benefit from rosiglitazone as an option to augment cardiorespiratory fitness.

\section{References}

1. O'Connor E, Kiely C, O'Shea D, Green S, Egana M. Similar level of impairment in exercise performance and oxygen uptake kinetics in middle-aged men and women with type 2 diabetes. American journal of physiology Regulatory, integrative and comparative physiology. 2012; 303(1): R70-6. doi: 10.1152/ajpregu.00012.2012
2. Regensteiner JG, Bauer TA, Huebschmann AG, et al. Sex differences in the effects of type 2 diabetes on exercise performance. Medicine and science in sports and exercise. 2015; 47(1): 58-65. doi: 10.1249/ MSS.0000000000000371.

3. Wei M, Gibbons LW, Kampert JB, Nichaman MZ, Blair SN. Low cardiorespiratory fitness and physical inactivity as predictors of mortality in men with type 2 diabetes. Annals of internal medicine. 2000; 132(8): 605-11.

4. Kokkinos P, Myers J, Nylen E, et al. Exercise capacity and all-cause mortality in African American and Caucasian men with type 2 diabetes. Diabetes care. 2009; 32(4): 623-8.doi: 10.2337/dc08-1876

5. Colberg SR, Albright AL, Blissmer BJ, et al. Exercise and type 2 diabetes: American College of Sports Medicine and the American Diabetes Association: joint position statement. Exercise and type 2 diabetes. Medicine and science in sports and exercise. 2010; 42(12): 2282-303. doi: 10.2337/dc10-1548.

6. Morrato EH, Hill JO, Wyatt HR, Ghushchyan V, Sullivan PW. Physical activity in U.S. adults with diabetes and at risk for developing diabetes, 2003. Diabetes care. 2007; 30(2): 203-9.

7. Thomas N, Alder E, Leese GP. Barriers to physical activity in patients with diabetes. Postgraduate medical journal. 2004; 80(943): 287-91.

8. Green S, Egana M, Baldi JC, Lamberts R, Regensteiner JG. Cardiovascular control during exercise in type 2 diabetes mellitus. Journal of diabetes research. 2015; 2015: 654204. doi: 10.1155/2015/654204.

9. Brunner-La Rocca HP, Weilenmann D, Follath F, et al. Oxygen uptake kinetics during low level exercise in patients with heart failure: relation to neurohormones, peak oxygen consumption, and clinical findings. Heart. 1999; 81(2): 121-7.

10. Kiely C, O'Connor E, O'Shea D, Green S, Egana M. Hemodynamic responses during graded and constant-load plantar flexion exercise in middle-aged men and women with type 2 diabetes. Journal of applied physiology. 2014; 117(7): 755-64. doi: 10.1152/ japplphysiol.00555.2014.

11. Aneja A, Tang WH, Bansilal S, Garcia MJ, Farkouh ME. Diabetic cardiomyopathy: insights into pathogenesis, diagnostic challenges, and therapeutic options. The American journal of medicine. 2008; 121(9): 748-57. doi: 10.1016/j.amjmed.2008.03.046.

12. Grewal J, McCully RB, Kane GC, Lam C, Pellikka PA. Left ventricular function and exercise capacity. JAMA : the journal of the American Medical Association. 2009; 301(3): 286-94. doi: 10.1001/ jama.2008.1022.

13. Poirier P, Garneau C, Bogaty P, et al. Impact of left ventricular diastolic dysfunction on maximal treadmill performance in normotensive subjects with well-controlled type 2 diabetes mellitus. The American journal of cardiology. 2000; 85(4): 473-7.

14. Fang ZY, Sharman J, Prins JB, Marwick TH. Determinants of exercise capacity in patients with type 2 diabetes. Diabetes care. 2005; 28(7): 1643-8.

15. Cardoso CR, Moraes RA, Leite NC, Salles GF. Relationships between reduced heart rate variability and pre-clinical cardiovascular disease in patients with type 2 diabetes. Diabetes research and clinical practice. 2014; 106(1): 110-7.doi: 10.1016/j.diabres.2014.07.005.

16. Gurdal A, Kasikcioglu E, Yakal S, Bugra Z. Impact of diabetes and diastolic dysfunction on exercise capacity in normotensive patients without coronary artery disease. Diabetes \& vascular disease research. 2015; 12(3): 181-8. doi: 10.1177/1479164114565631 
17. Sacre JW, Jellis CL, Jenkins C, et al. A six-month exercise intervention in subclinical diabetic heart disease: effects on exercise capacity, autonomic and myocardial function. Metabolism: clinical and experimental. 2014; 63(9): 1104-14. doi: 10.1016/j. metabol.2014.05.007.

18. Alves AJ, Ribeiro F, Goldhammer E, et al. Exercise training improves diastolic function in heart failure patients. Medicine and science in sports and exercise. 2012; 44(5): 776-85.doi: 10.1249/ MSS.0b013e31823cd16a.

19. Reusch JE, Bridenstine M, Regensteiner JG. Type 2 diabetes mellitus and exercise impairment. Reviews in endocrine \& metabolic disorders. 2013; 14(1): 77-86. doi: 10.1007/s11154-012-9234-4.

20. Byrkjeland R, Edvardsen E, Njerve IU, Arnesen H, Seljeflot I, Solheim S Insulin levels and HOMA index are associated with exercise capacity in patients with type 2 diabetes and coronary artery disease. Diabetology \& metabolic syndrome. 2014; 6(1): 36. doi: 10.1186/1758-5996-6-36.

21. Fontes-Carvalho R, Ladeiras-Lopes R, Bettencourt P, Leite-Moreira A, Azevedo A. Diastolic dysfunction in the diabetic continuum: association with insulin resistance, metabolic syndrome and type 2 diabetes. Cardiovascular diabetology. 2015; 14: 4.doi: 10.1186/ s12933-014-0168-x.

22. Tousoulis D, Papageorgiou N, Androulakis E, et al. Diabetes mellitusassociated vascular impairment: novel circulating biomarkers and therapeutic approaches. Journal of the American College of Cardiology. 2013; 62(8): 667-76.doi: 10.1016/j.jacc.2013.03.089.

23. Manucha W, Ritchie B, Ferder L. Hypertension and insulin resistance: implications of mitochondrial dysfunction. Current hypertension reports. 2015; 17(1): 504.doi: 10.1007/s11906-014-0504-2.

24. Mandavia CH, Pulakat L, DeMarco V, Sowers JR. Over-nutrition and metabolic cardiomyopathy. Metabolism: clinical and experimental. 2012; 61(9): 1205-10. doi: 10.1016/j.metabol.2012.02.013.

25. Carnethon MR, Jacobs DR, Jr., Sidney S, Liu K, study C. Influence of autonomic nervous system dysfunction on the development of type 2 diabetes: the CARDIA study. Diabetes care. 2003; 26(11): 3035-41.

26. Myers J, Prakash M, Froelicher V, Do D, Partington S, Atwood JE. Exercise capacity and mortality among men referred for exercise testing. The New England journal of medicine. 2002; 346(11): 793801.

27. Church TS, LaMonte MJ, Barlow CE, Blair SN. Cardiorespiratory fitness and body mass index as predictors of cardiovascular disease mortality among men with diabetes. Archives of internal medicine. 2005; 165(18): 2114-20.

28. Bacchi E, Negri C, Zanolin ME, et al. Metabolic effects of aerobic training and resistance training in type 2 diabetic subjects: a randomized controlled trial (the RAED2 study). Diabetes care. 2012; 35(4): 67682. doi: $10.2337 /$ dc11-1655.

29. Boule NG, Kenny GP, Haddad E, Wells GA, Sigal RJ. Meta-analysis of the effect of structured exercise training on cardiorespiratory fitness in Type 2 diabetes mellitus. Diabetologia. 2003; 46(8): 1071-81.

30. Simmons RK, van Sluijs EM, Hardeman W, Sutton S, Griffin SJ ProActive project t. Who will increase their physical activity? Predictors of change in objectively measured physical activity over 12 months in the ProActive cohort. BMC public health. 2010; 10: 226. doi: 10.1186/1471-2458-10-226.

31. Bames P, Schoenborn C. Trends in adults receiving a recommendation for exercise or other physical activity from a physician or other health professional. US Department of Health and Human Services, Centers for Disease Control and Prevention, National Center for Health Statistics. 2012

32. Regensteiner JG, Bauer TA, Reusch JE. Rosiglitazone improves exercise capacity in individuals with type 2 diabetes. Diabetes care. 2005; 28(12): 2877-83.

33. Kadoglou NP, Iliadis F, Sailer N, et al. Exercise training ameliorates the effects of rosiglitazone on traditional and novel cardiovascular risk factors in patients with type 2 diabetes mellitus. Metabolism: clinical and experimental. 2010; 59(4): 599-607. doi: 10.1016/j. metabol.2009.09.002.

34. Takada S, Hirabayashi K, Kinugawa S, et al. Pioglitazone ameliorates the lowered exercise capacity and impaired mitochondrial function of the skeletal muscle in type 2 diabetic mice. European journal of pharmacology. 2014; 740: 690-6. doi: 10.1016/j.ejphar.2014.06.008.

35. Pagel-Langenickel I, Schwartz DR, Arena RA, et al. A discordance in rosiglitazone mediated insulin sensitization and skeletal muscle mitochondrial content/activity in Type 2 diabetes mellitus. American journal of physiology Heart and circulatory physiology. 2007; 293(5): H2659-66.

36. Sixt S, Rastan A, Desch S, et al. Exercise training but not rosiglitazone improves endothelial function in prediabetic patients with coronary disease. European journal of cardiovascular prevention and rehabilitation : official journal of the European Society of Cardiology, Working Groups on Epidemiology \& Prevention and Cardiac Rehabilitation and Exercise Physiology. 2008; 15(4): 473-8. doi: 10.1097/HJR.0b013e3283002733.

37. Petersen KF, Shulman GI. Etiology of insulin resistance. The American journal of medicine. 2006; 119(5 Suppl 1): S10-6.

38. Rojas E, Rodriguez-Molina D, Bolli P, et al. The role of adiponectin in endothelial dysfunction and hypertension. Current hypertension reports. 2014; 16(8): 463. doi: 10.1007/s11906-014-0463-7.

39. Kadoglou NP, Iliadis F, Angelopoulou N, Perrea D, Liapis CD, Alevizos M. Beneficial effects of rosiglitazone on novel cardiovascular risk factors in patients with Type 2 diabetes mellitus. Diabetic medicine : a journal of the British Diabetic Association. 2008; 25(3): 333-40. doi: 10.1111/j.1464-5491.2007.02375.x.

40. Braun B, Eze P, Stephens BR, et al. Impact of metformin on peak aerobic capacity. Applied physiology, nutrition, and metabolism = Physiologie appliquee, nutrition et metabolisme. 2008; 33(1): 617. doi: $10.1139 / \mathrm{H} 07-144$.

41. Johnson ST, Robert C, Bell GJ, Bell RC, Lewanczuk RZ, Boule NG. Acute effect of metformin on exercise capacity in active males. Diabetes, obesity \& metabolism. 2008; 10(9): 747-54.

42. Boule NG, Kenny GP, Larose J, Khandwala F, Kuzik N, Sigal RJ. Does metformin modify the effect on glycaemic control of aerobic exercise, resistance exercise or both? Diabetologia. 2013; 56(11): 2378-82. doi: 10.1007/s00125-013-3026-6. 